\title{
The Running Wheel Enhances Food Anticipatory Activity: An Exploratory Study
}

\author{
Danilo E. F. L. Flôres ${ }^{1,2}$, Crystal N. Bettilyon ${ }^{1}$, Lori Jia ${ }^{1,3}$ and Shin Yamazaki ${ }^{1 *}$ \\ ${ }^{1}$ Department of Neuroscience, University of Texas Southwestern Medical Center, Dallas, TX, USA, ${ }^{2}$ Institute of Biosciences, \\ University of São Paulo, São Paulo, Brazil, ${ }^{3}$ Hockaday School, Dallas, TX, USA
}

Rodents anticipate rewarding stimuli such as daily meals, mates, and stimulant drugs. When a single meal is provided daily at a fixed time of day, an increase in activity, known as food anticipatory activity (FAA), occurs several hours before feeding time. The factors affecting the expression of FAA have not been well-studied. Understanding these factors may provide clues to the undiscovered anatomical substrates of food entrainment. In this study we determined whether wheel-running activity, which is also rewarding to rodents, modulated the robustness of FAA. We found that access to a freely rotating wheel enhanced the robustness of FAA. This enhancement was lost when the wheel was removed. In addition, while prior exposure to a running wheel alone did not enhance FAA, the presence of a locked wheel did enhance FAA as long as mice had previously run in the wheel. Together, these data suggest that FAA, like wheel-running activity, is influenced by reward signaling.

\section{OPEN ACCESS}

Edited by:

John D. Salamone,

University of Connecticut, USA

Reviewed by:

Alicia Izquierdo,

University of California, Los Angeles,

USA

Hirofumi Morishita,

Mount Sinai School of Medicine, USA

*Correspondence:

Shin Yamazak

shin.yamazaki@utsouthwestern.edu

Received: 06 April 2016 Accepted: 22 June 2016 Published: 05 July 2016

Citation:

Flôres DEFL, Bettilyon CN, Jia L and Yamazaki S (2016) The Running

Wheel Enhances Food Anticipatory Activity: An Exploratory Study. Front. Behav. Neurosci. 10:143. doi: 10.3389/fnbeh.2016.00143
Keywords: circadian, mouse, reward, restricted feeding, food-entrainable oscillator

\section{INTRODUCTION}

Organisms use circadian clocks to anticipate daily changes in the environment (temperature, food availability, and predation). It has been suggested that this anticipation is critical for survival (Antle and Silver, 2009; DeCoursey, 2014; Spoelstra et al., 2016). For proper anticipation clocks must retain a stable phase-relationship with, or entrain to, daily environmental cycles (Winfree, 1980; Johnson et al., 2003). Light is the primary environmental signal that entrains a master circadian pacemaker located in the suprachiasmatic nucleus (SCN; Moore and Eichler, 1972; Stephan and Zucker, 1972; Rusak, 1977). The SCN orchestrates an ensemble of rhythms among the peripheral circadian clocks located in most peripheral organs (Yamazaki et al., 2000; Yoo et al., 2004; Izumo et al., 2014) and controls circadian rhythms in physiology and behavior (Moore and Eichler, 1972; Sawaki et al., 1984; Lehman et al., 1987; Ralph et al., 1990). The molecular machinery that generates these circadian rhythms in the SCN and peripheral clocks has been extensively characterized (Ko and Takahashi, 2006). Daily food availability is another signal that can entrain circadian clocks. In laboratory studies, locomotor activity of rodents entrains to restricted food availability, and the rodents become active several hours before food is presented (Richter, 1922). This so-called food anticipatory activity (FAA) is controlled by a circadian pacemaker, the food-entrainable oscillator (Mistlberger, 1994; Stephan, 2002). SCN-ablated animals still exhibit normal FAA, indicating that the food-entrainable oscillator is located outside of the SCN (Mistlberger, 1994; Stephan, 2002). However, despite exhaustive attempts to identify the food-entrainable oscillator, its anatomical locus has yet to be discovered (Davidson, 2009). 
It has also been shown that the food-entrainable oscillator does not rely on the canonical molecular circadian timekeeping mechanism (Pitts et al., 2003; Iijima et al., 2005; Pendergast et al., 2009, 2012; Storch and Weitz, 2009; Flores et al., 2016). Although the circadian properties of food entrainment have been elucidated, the factors affecting the expression of FAA have not been well-studied. Understanding factors that regulate the robustness of FAA may provide clues toward identifying the anatomical locus of the food-entrainable circadian oscillator.

Several lines of evidence suggest the reward system is involved in FAA expression (Webb et al., 2009). In addition to anticipating restricted food availability, rodents also anticipate other daily rewarding stimuli, such as palatable meals, females in estrous, and stimulant drugs (Mistlberger and Rusak, 1987; Mendoza et al., 2005a,c; Verwey et al., 2007; Angeles-Castellanos et al., 2008; Hsu et al., 2010a,b; Jansen et al., 2012; Landry et al., 2012; Keith et al., 2013; Mohawk et al., 2013; Flores et al., 2016). Recent studies show that dopamine and opioids, which are important components of the reward pathway, regulate the robustness of FAA (Kas et al., 2004; Mendoza and Challet, 2014). FAA is attenuated in D1 receptor, but not D2 receptor, knockout mice (Gallardo et al., 2014; Michalik et al., 2015). Systemic pretreatment (before the onset of food) with D1 and D2 receptor antagonists attenuated FAA in ICR mice (Liu et al., 2012). It has also been shown that mu-opioid receptor knockout mice show diminished FAA (Kas et al., 2004). Together these data suggest that dopamine and endogenous opioids can alter the robustness of FAA. Wheel-running is rewarding to rodents and is known to activate the dopaminergic system and influence endogenous opioids in their brains (Sherwin, 1998; Novak et al., 2012; Morgan et al., 2015). In this study, we investigate the effect of the running wheel on the robustness of FAA in C57BL/6J mice.

\section{MATERIALS AND METHODS}

\section{Animals}

Wild-type C57BL/6J male mice ( $n=27,4-10$ weeks of age) were obtained from either the E. K. Wakeland Mouse Breeding Core (UT Southwestern, Dallas, TX, USA) or our breeding colony at UT Southwestern. After weaning, mice were grouphoused with ad libitum access to chow (Teklad Global 18\% Protein Rodent Diet 2918; Harlan, Madison, WI, USA) and water in cages without running wheels. The $12 \mathrm{~h}$ light: $12 \mathrm{~h}$ dark (12L:12D) cycle in the holding room was generated by fluorescent bulbs. All experiments were carried out in accordance with the National Institutes of Health Guidelines regarding the care and use of animals for experimental procedures and were approved by the Institutional Animal Care and Use Committee at UT Southwestern Medical Center (Protocol \#: 2013-0035).

\section{Activity Recording}

Animals were singly housed in cages with ad libitum access to water in light-tight ventilated boxes $\left(22-23^{\circ} \mathrm{C}, 19-54 \%\right.$ relative humidity) in $18 \mathrm{~L}: 6 \mathrm{D}$ or $12 \mathrm{~L}: 12 \mathrm{D}$. Light was generated by white LEDs $\left(40 \mu \mathrm{W} / \mathrm{cm}^{2} / \mathrm{s}, 220\right.$ lux inside the cage) or green LEDs $\left(7 \mu \mathrm{W} / \mathrm{cm}^{2} / \mathrm{s}, 55\right.$ lux inside the cage); specified for each experiment (see figure legends). Mice were housed either in cages with running wheels (length $\times$ width $\times$ height: $29.5 \times 11.5 \times 12.0 \mathrm{~cm}$; wheel diameter $11.0 \mathrm{~cm})$ or without running wheels $(29.5 \times 11.5 \times 12.0 \mathrm{~cm}$ or $28.5 \times 16.5 \times 13.0 \mathrm{~cm})$. In experiments with locked wheels, a clip was used to prevent the wheel from rotating. The number of wheel revolutions was monitored by a micro-switch and general activity was monitored with a passive infrared sensor (product ID 189, Adafruit, New York City, NY, USA) placed above the cage. Activity was continuously recorded every minute using the ClockLab system (Actimetrics, Wilmette, IL, USA). Cages and water bottles were changed at least once every 3 weeks.

\section{Restricted Feeding}

In both 18L:6D and 12L:12D, food was initially removed $2 \mathrm{~h}$ before lights-off and left out for $16 \mathrm{~h}$. For 2 days, chow was provided for $8 \mathrm{~h}$ (from $10 \mathrm{~h}$ before lights-off to $2 \mathrm{~h}$ before lights-off), and then for $6 \mathrm{~h}$ (from $10 \mathrm{~h}$ before light-off to $4 \mathrm{~h}$ before lights-off) for the subsequent 2 days. Thereafter, chow was provided for $4 \mathrm{~h}$ (from $8 \mathrm{~h}$ before lights-off to $4 \mathrm{~h}$ before lights-off). Food was manually placed on the bottom of the cage and/or in the food hopper. When food was removed, the bottom of the cage was carefully inspected to remove any remaining small pieces of chow.

\section{Data Analysis}

Activity was double-plotted in actograms (6-min bins; normalized format, ClockLab). Actograms (wheel-running and general activity) for all individual mice are provided in the Supplementary Material. Twenty-four hour individual average activity profiles were used to quantify the robustness of FAA. Using ClockLab, individual average activity profiles (6-min bins) were generated from activity data for the last 5 or 7 days (specified in the figure legends) in each wheel condition. The robustness of FAA was quantified by measuring the area under the curve of the bout of activity that occurred prior to the onset of food availability (Supplementary Figure 1). The area under the curve was measured by free-hand tracing using the ImageJ software (National Institutes of Health). The twenty-four hour group average profiles are the average of the individual activity profiles.

\section{Statistics}

The summary of the statistics used is presented in Supplementary Table 1. The robustness of FAA (FAA AUC) was compared among the different cage types in each experiment. Because the experimental conditions in each experiment were slightly different, we did not compare FAA between experiments. The data for each cage type was tested for normality using the Shapiro-Wilk test. When data had no normal distribution, a non-parametric test was used. Either the paired two-tailed 
$t$-test or Wilcoxon signed-rank test was used for experiments with two conditions. ANOVA with repeated measures was used for experiments with three conditions, and if significance was detected a post hoc multiple comparison test (Tukey's) was used. The FAA AUC measure for animal \#206 in the Locked Wheel cage (2757) was identified beyond the Quartile $3\left(\mathrm{Q}_{3}\right)+1.5 \times$ Interquartile Range (IQR). Therefore, \#206 was excluded from the statistical analysis. The software PAST version 3.08 (Hammer et al., 2001) was used for all statistical analyses. Criteria $(p<0.05)$ was used for statistical significance.

\section{RESULTS}

\section{Wheel-Running Enhances Food Anticipatory Activity}

We first determined whether wheel running activity affects FAA (Figures 1A-C). We housed mice in cages without running wheels (Figure 1A, no wheel ad libitum) and then performed daytime restricted feeding. Weak FAA was present during restricted feeding without a running wheel (Figures 1A-C, no wheel). After 8 days of stable $4 \mathrm{~h}$ restricted feeding, we moved mice to cages with running wheels and continued restricted feeding. The robustness of FAA was increased by the introduction of the running wheel (Figures 1A-C, free wheel; except one mouse shown in Figure 1B). The effect of the running wheel extinguished upon moving the mice to cages without running wheels, evidenced by the decrease in FAA robustness back to prewheel levels (Figures 1A-C, no wheel 2). These data show that the robustness of FAA was enhanced by running wheel activity, but this enhancement was lost when the wheel was removed.

\section{Prior Wheel-Running Experience Alone does not Enhance FAA}

We next determined whether prior wheel-running experience affected the robustness of FAA (Figures 1D-F). We first housed mice in cages with running wheels and provided chow ad libitum (Figure 1D, free wheel ad libitum). On day 13, we moved the mice to cages without wheels and performed restricted feeding (Figures 1D-F, no wheel). The mice expressed weak FAA that was enhanced when the mice were subsequently moved to cages with running wheels (Figures 1D-F, free wheel). Then, the robustness of FAA decreased again when the mice were moved back to cages without wheels (Figures 1D-F, no wheel 2). This study shows that prior experience of wheel-running alone did not enhance FAA.

\section{Naïve Exposure to a Locked Running Wheel Alone does not Enhance FAA}

We next tested if the presence of a locked wheel in the cage enhances FAA (Figure 2). Consistent with our previous results, when we performed restricted feeding in mice housed in cages without wheels, the mice had weak FAA (Figure 2, no wheel). On day 29, we moved the mice to cages with locked wheels and continued restricted feeding. In the presence of the locked wheel, FAA remained weak (Figure 2, locked wheel). To determine if the free wheel had an enhancement effect on this cohort, we next unlocked the wheels (Figure 2, free wheel). As before, the robustness of FAA was enhanced with rotating running wheels, and FAA returned to low levels (similar to the locked wheel condition) when the wheels were removed (Figure 2, no wheel 2). These data demonstrate that the presence of a locked running wheel alone did not enhance FAA.

\section{Prior Wheel-Running Experience Combined with a Locked Wheel Enhances FAA}

Next we tested if previous wheel-running experience affected FAA in the presence of a locked wheel (Figure 3). We housed mice with freely rotating wheels (Figures $\mathbf{3 A}-\mathbf{C}$, free wheel ad libitum) and performed restricted feeding (Figures $3 \mathrm{~A}-\mathrm{C}$, free wheel). The mice developed robust FAA. When the wheels were locked, the robustness of FAA was unchanged (Figures 3A-C, locked wheel). FAA stayed robust when the wheels were unlocked again (Figures $\mathbf{3 A}-\mathbf{C}$, free wheel 2). Interestingly, though, after an intervening exposure to free wheel (and enhancement of FAA), mice were placed in cages with no wheels and the robustness of FAA decreased to levels lower than the locked wheel condition (Figures 3A-C, no wheel). These data demonstrate that after prior wheel-running, locked wheels were able to enhance the robustness of FAA.

In the previous experiment, FAA was established in the presence of freely rotating wheels. Next we exposed mice to free wheels during ad libitum feeding (Figure 3D, free-wheel ad libitum), but then started restricted feeding after the running wheels were locked (Figures 3D-F, locked wheel). The mice developed robust FAA in this paradigm even though restricted feeding was performed only in the presence of locked wheels. When wheels were then unlocked, FAA remained robust but did not increase further (Figures 3D-F, free wheel). FAA remained robust upon locking the wheels again (Figures 3D-F, locked wheel 2). In contrast, when the mice were subsequently moved to cages without wheels, the robustness of FAA decreased (Figures 3D-F, no wheel). Together these data show that locked wheels enhanced FAA, as long as the mice previously participated in wheel-running activity. These findings should be verified in future studies with additional mice.

\section{Photoperiod Alters the Robustness of FAA}

Previous studies have shown that dopamine signaling is influenced by photoperiod (Sorg et al., 2011; Deats et al., 2015; Goda et al., 2015). We previously found that FAA is more robust in long photoperiods (18L:6D) compared to short photoperiods (12L:12D) (Pendergast et al., 2009). To test whether there was a synergistic effect of long photoperiod and running wheel activity on the robustness of FAA, we performed restricted feeding in 12L:12D to contrast our results in 18L:6D. All mice were initially housed with free wheels and fed ad libitum in 12L:12D (Figures 4A,D, free wheel 
A

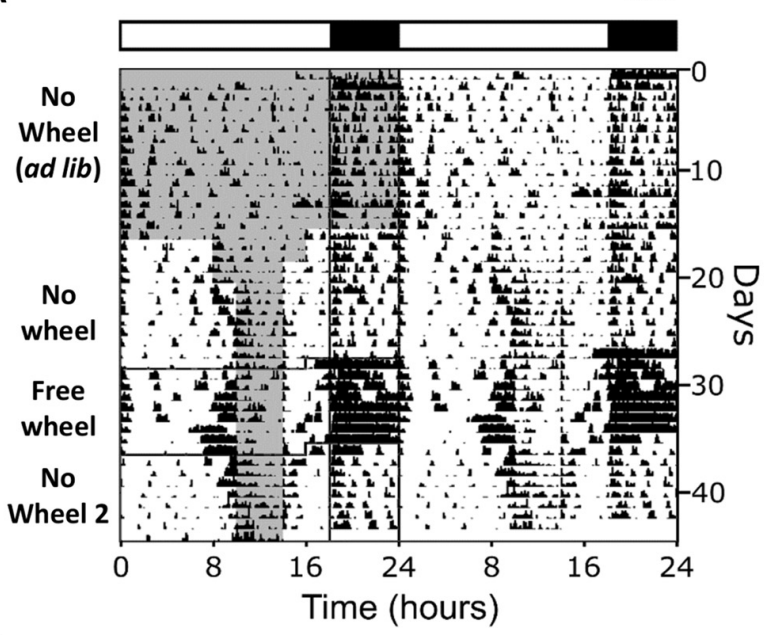

B

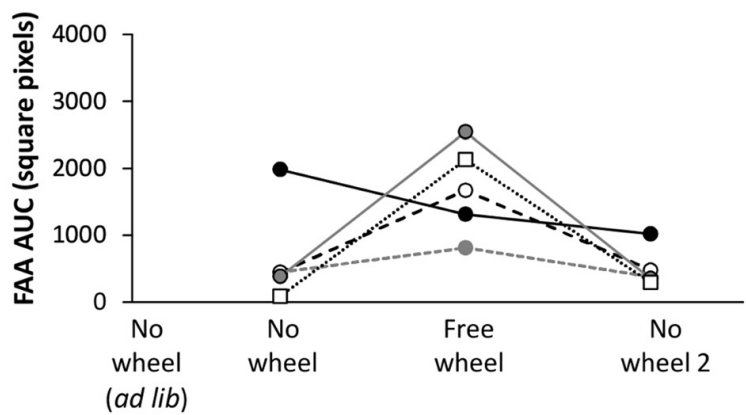

C

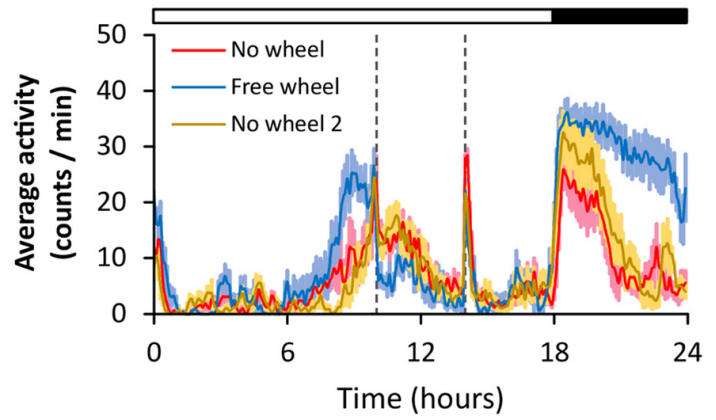

D

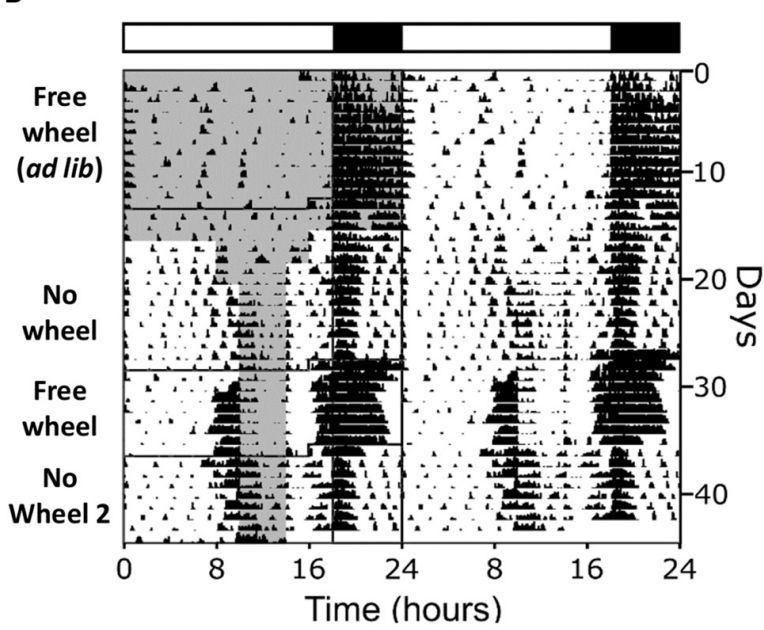

E

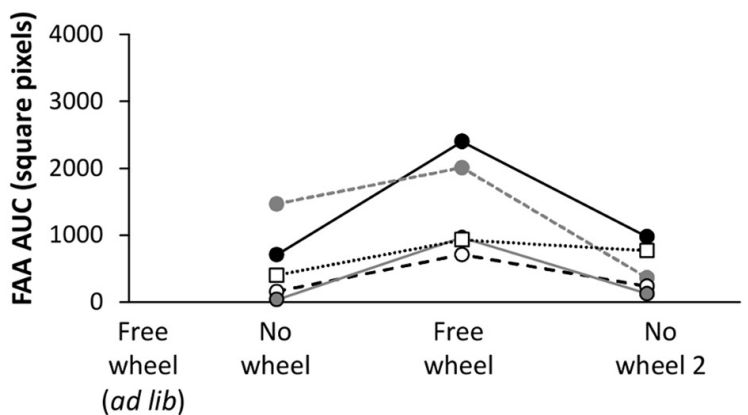

F

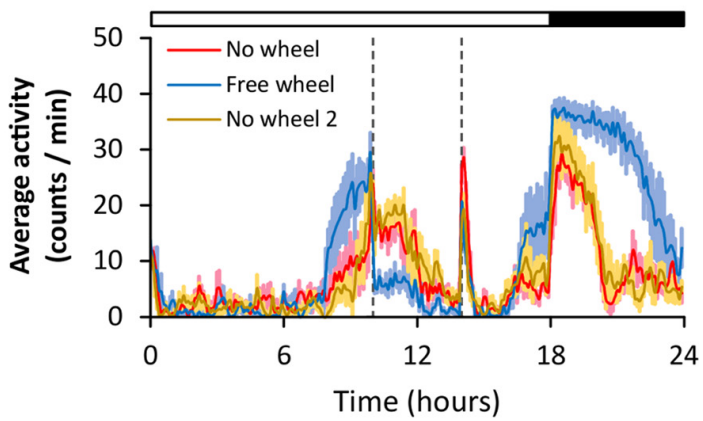

FIGURE 1 | Wheel-running enhances food anticipatory activity (FAA) irrespective of prior wheel running experience. FAA from wheel-naïve (A-C; $n=5)$ and wheel-exposed (D-F; $n=5$ ) mice was measured in 18L:6D (white LEDs). During restricted feeding, chow was manually placed and removed from the food hopper. The times when food was available are shown as gray shading on the left half of each representative double-plotted actogram (A,D; white and black bars show light and dark, respectively). FAA was quantified (AUC of the last 5 days of data) for each mouse (B,E; unique symbols connected by lines) in successive no-wheel, free-wheel, and then no-wheel conditions. Twenty-four hour group average activity profiles (C,F; dotted vertical lines indicate the time of food availability) show the mean (solid line) and standard deviation (light colored area). Time 0 is lights on. Cage dimensions: no-wheel: $29.5 \times 11.5 \times 12.0 \mathrm{~cm}$; free-wheel: $29.5 \times 11.5 \times 12.0 \mathrm{~cm}$ with an $11.0 \mathrm{~cm}$ diameter running wheel. All individual actograms and activity profiles are shown in the Supplementary Figures $2-4$.

ad libitum). We then gave the mice either free wheels or locked wheels and performed restricted feeding in 12L:12D (Figure 4, free wheel, locked wheel). Mice in both conditions developed FAA. Because all mice initially participated in wheel-running activity, the robustness of FAA was similar in mice with free wheels and locked wheels (Figures 4A-F). However, FAA was less robust in 12L:12D compared to 18L:6D. 

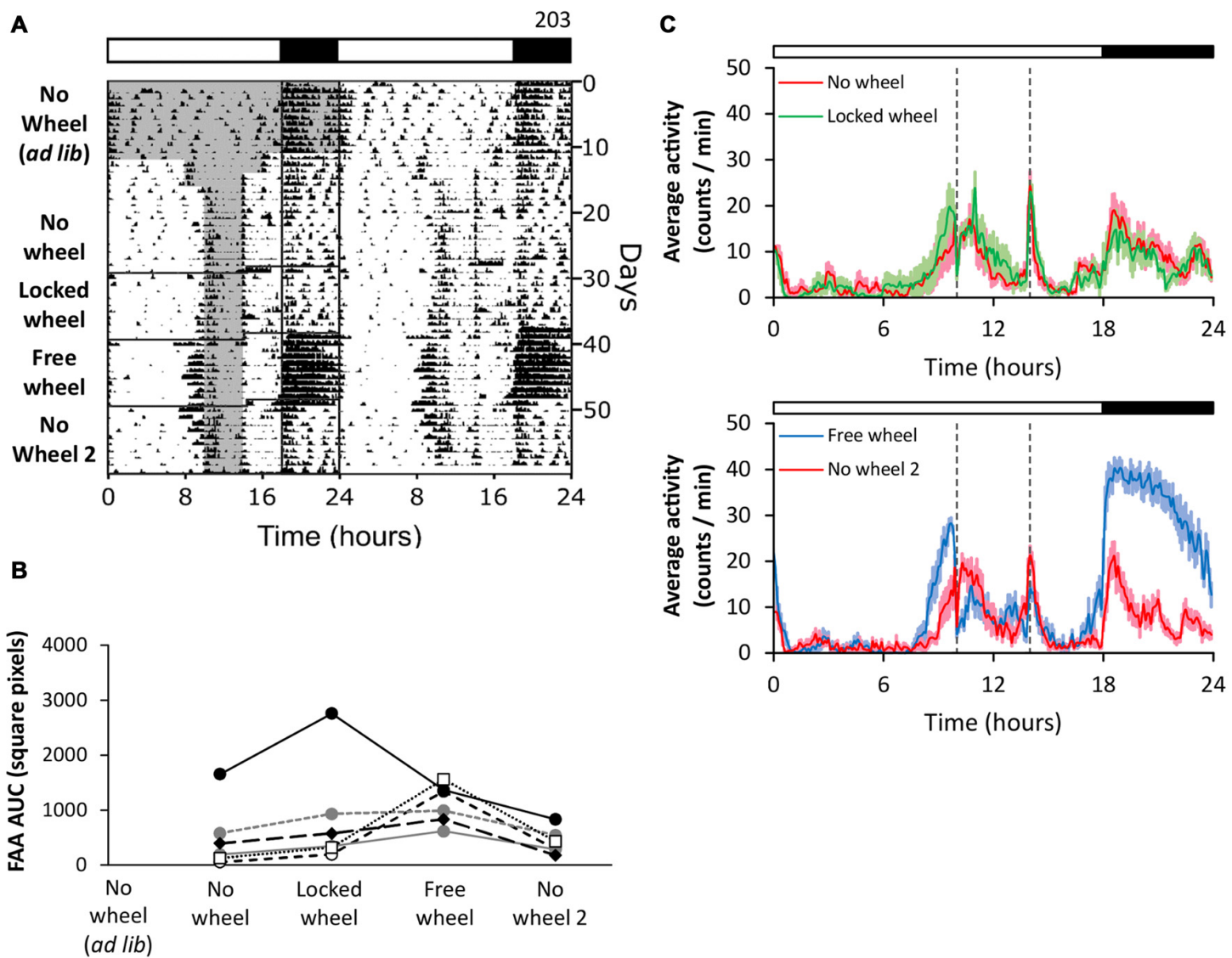

FIGURE 2 | Naïve exposure to a locked running wheel alone does not enhance FAA. FAA from wheel-naïve mice $(n=6)$ was measured in 18L:6D (green LEDs). During restricted feeding, chow was manually placed and removed from the food hopper and cage bottom. The times when food was available are shown as gray shading on the left half of the representative double-plotted actogram (A; white and black bars show light and dark, respectively). FAA was quantified (AUC of the last 7 days of data) for each mouse (B; unique symbols connected by lines) in successive no-wheel, locked-wheel, free-wheel, and no-wheel conditions. Twenty-four hour group average activity profiles (C) dotted vertical lines indicate the time of food availability) show the mean (solid line) and standard deviation (light colored area). Time 0 is lights on. Cage dimensions: no-wheel: $28.5 \times 16.5 \times 13.0 \mathrm{~cm}$; with-wheel (locked and free): $29.5 \times 11.5 \times 12.0 \mathrm{~cm}$ with an $11.0 \mathrm{~cm}$ diameter running wheel. All individual actograms and activity profiles are shown in the Supplementary Figures 5-7.

It was possible that the reduction in robustness of FAA in 12L:12D compared to $18 \mathrm{~L}: 6 \mathrm{D}$ was due to different phases of food availability relative to the light-dark cycle. Thus, we next shifted the light-dark cycle relative to the time of restricted feeding while mice stayed in their respective free- or lockedwheel conditions in 12L:12D. We shifted the light-dark cycle four times to achieve restricted feeding at three phases: late day (ZT7-11), mid-day (ZT4-8), and early day (ZT1-5). Even though FAA was more robust during late day and mid-day restricted feeding compared to early day feeding, FAA was never as robust as in 18L:6D (Figures 4A,B,D,E). Therefore, the phase of restricted feeding did not account for the reduced robustness of FAA in 12L:12D.

\section{Nocturnal Activity During Restricted Feeding is Increased in Wheel-Running Conditions}

In all experiments in which mice were housed with freely rotating wheels during restricted feeding, nighttime activity increased
(Figures 1C,F, 2C, 3C,F, 4C,F). However, nocturnal activity did not increase in cages with locked wheels even if mice had been previously exposed to wheel-running (Figures 3C,F). These data suggest that FAA (output of the food-entrainable oscillator) and nocturnal activity (output of the SCN) are modulated by different mechanisms.

\section{DISCUSSION}

Voluntary wheel-running is not only physical exercise but also a highly rewarding stimulus for many animal species (Sherwin, 1998; Novak et al., 2012; Morgan et al., 2015). Rodents ran greater distances in wheels than on treadmills (Sherwin, 1998). Moreover, rodents worked to unlock running wheels (Kagan and Berkun, 1954; Collier and Hirsch, 1971; Iversen, 1993; Belke, 1997) and developed conditioned place preferences for contexts associated with wheel-running (Belke and Wagner, 2005). Numerous studies have shown that voluntary wheel-running induced molecular and neuronal changes in the brainstem, hypothalamus, and basal ganglia 

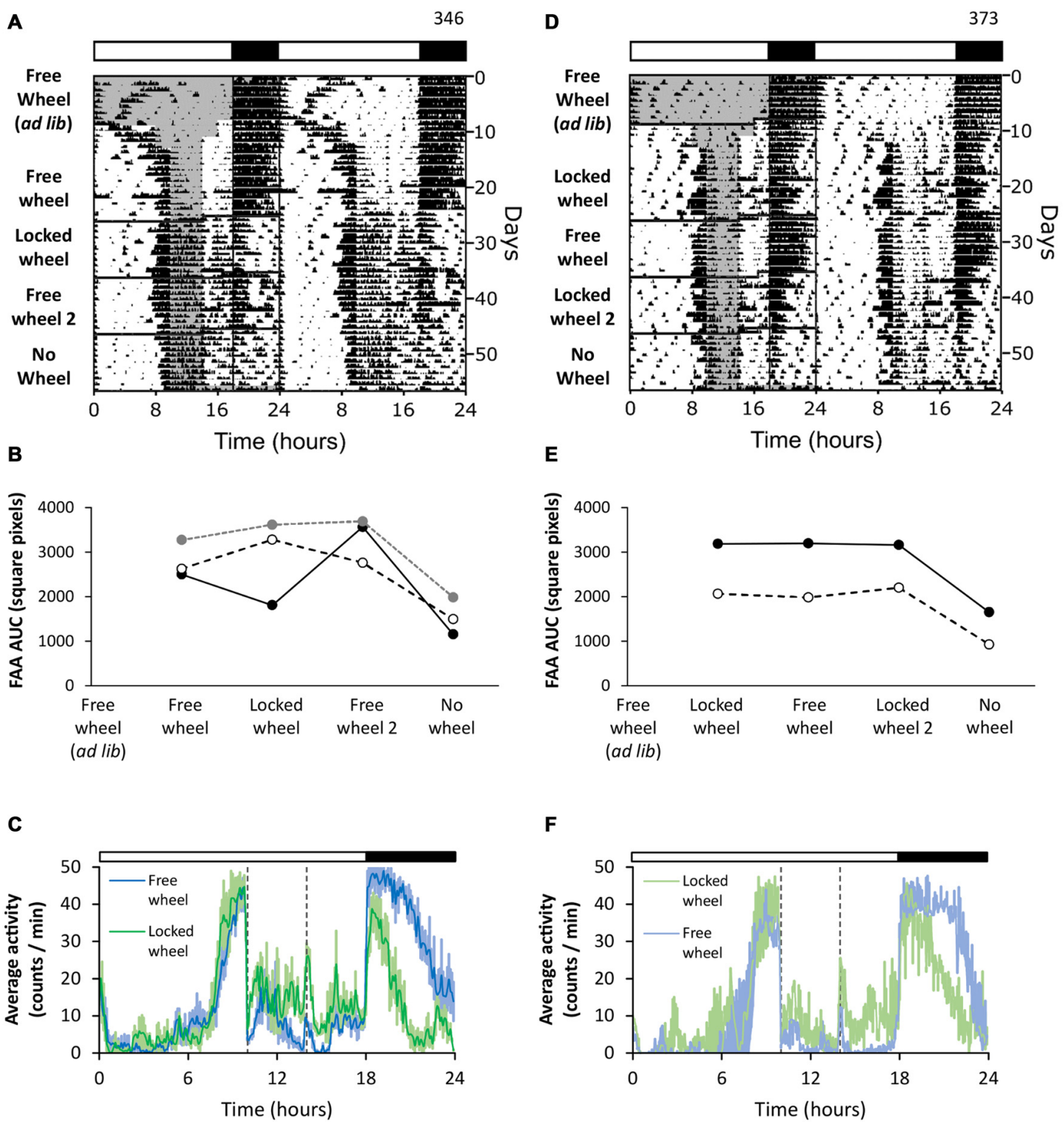

$\mathbf{F}$
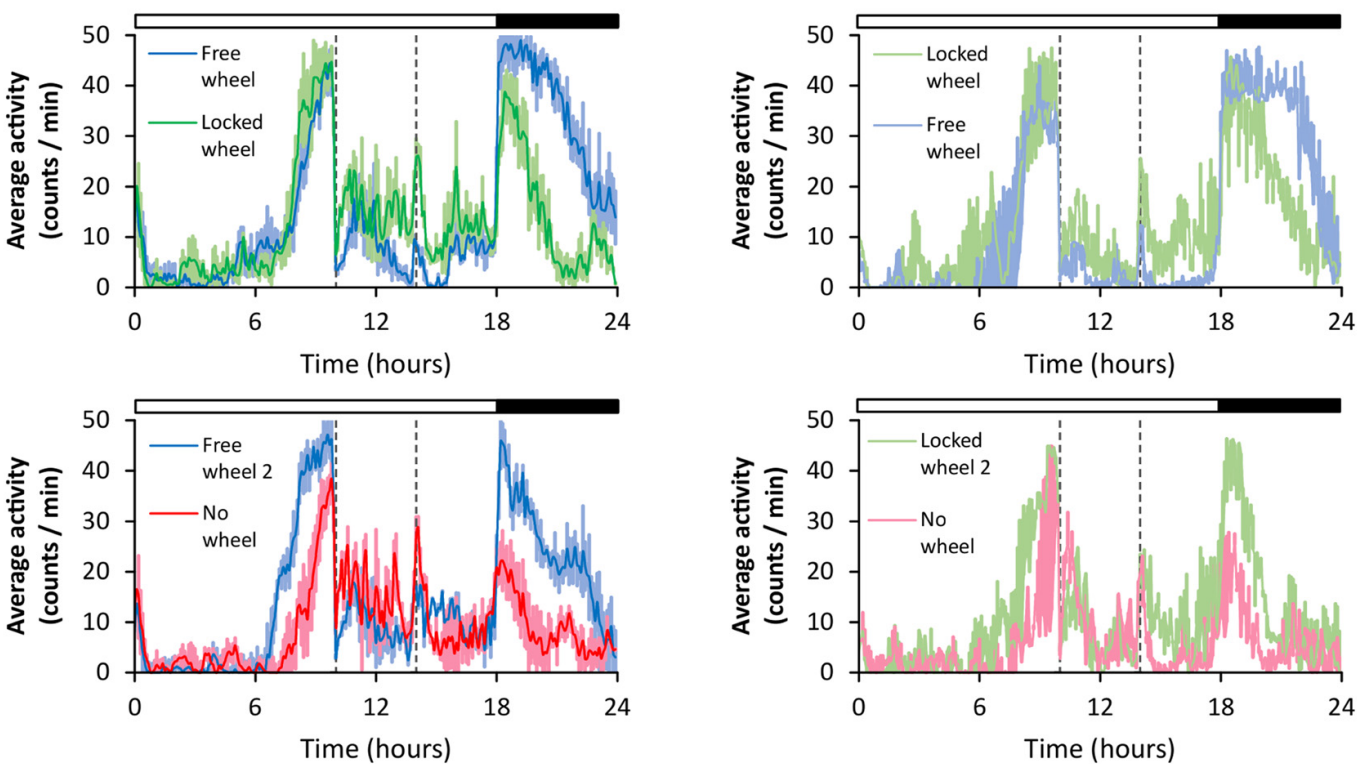

FIGURE 3 | Prior wheel-running experience combined with a locked wheel enhances FAA. Mice were first exposed to running wheels in 18L:6D (green LEDs). Restricted feeding was initiated in either free-wheel (A-C; $n=3$ ) or locked-wheel (D-F; $n=2)$ conditions. During restricted feeding, chow was manually placed and removed from the food hopper and cage bottom. In (B), FAA was quantified (AUC of the last 7 days of data) for each mouse (unique symbols connected by lines) in successive free-wheel, locked-wheel, free-wheel, and no-wheel conditions. In (E), FAA was quantified (AUC of the last 7 days of data) for each mouse (unique symbols connected by lines) in successive locked-wheel, free-wheel, locked-wheel, no-wheel conditions. Twenty-four hour group average activity profiles (C,F; dotted vertical lines indicate the time of food availability) show the mean (solid line) and standard deviation (C) or range (F) with light colored areas. Time 0 is lights on. Cage dimensions: no-wheel: $28.5 \times 16.5 \times 13.0 \mathrm{~cm}$; with-wheel (locked and free): $29.5 \times 11.5 \times 12.0 \mathrm{~cm}$ with an $11.0 \mathrm{~cm}$ diameter running wheel. All individual actograms and activity profiles are shown in the Supplementary Figures 8-10. 


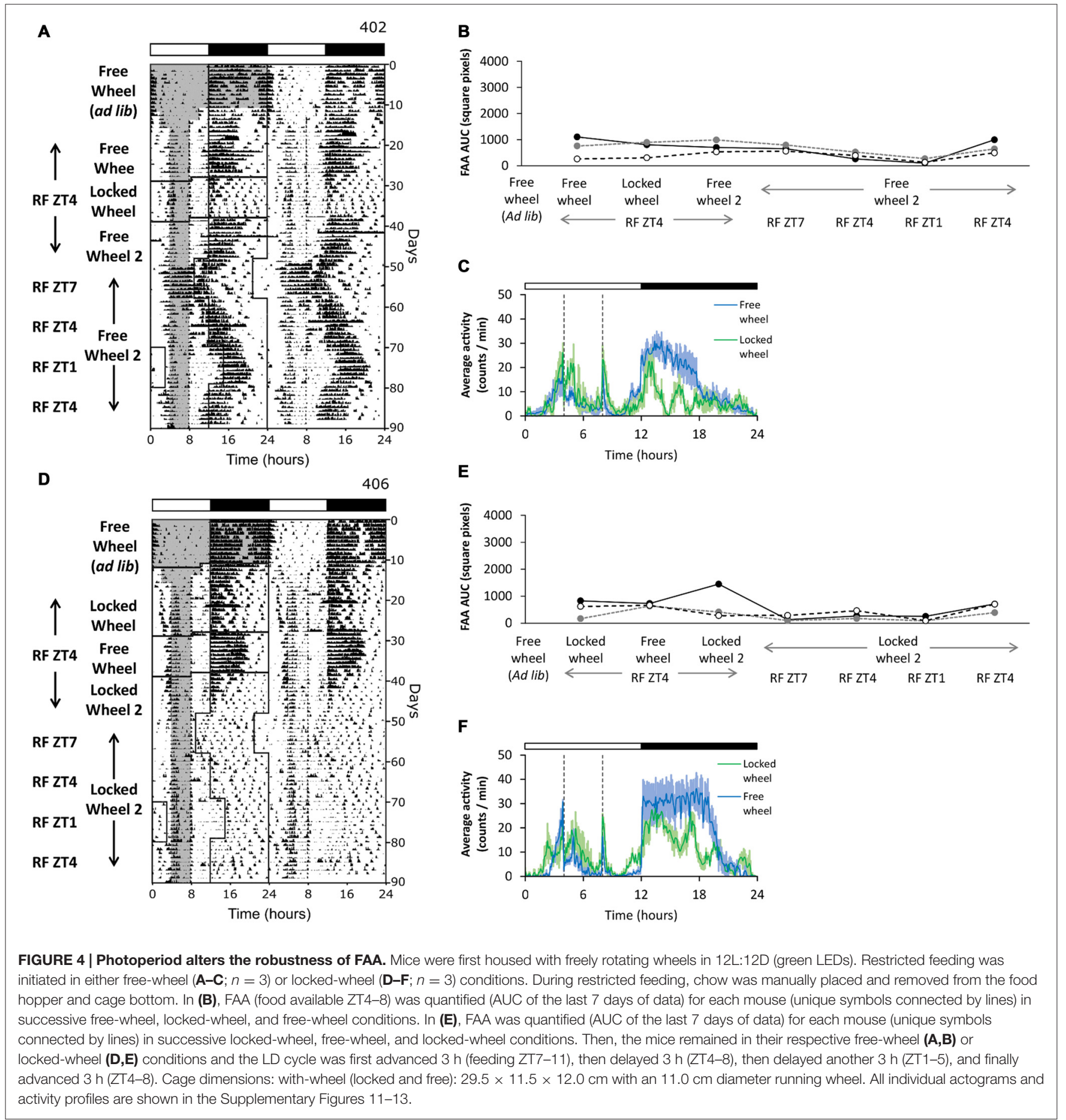

(Morgan et al., 2015). These responses are not limited to rodents in laboratory settings; wheels placed in the natural environment were readily used by wild animals (Meijer and Robbers, 2014).

Although FAA in rodents has been primarily studied in the laboratory, it has been suggested that it represents an activated foraging drive (Mather, 1981). Temporal organization of foraging behaviors is likely critical for the survival of many species (e.g., to increase chances of obtaining food while evading predation). Thus, one can speculate that associating foraging with reward ensures that animals are highly motivated to perform this behavior. Support for this hypothesis comes from several studies linking the dopaminergic reward system to FAA expression (Webb et al., 2009; Mendoza and Challet, 2014). For example, FAA was attenuated when the core of the nucleus accumbens was chemically lesioned in rats (Mendoza et al., 2005b). In addition, FAA was attenuated by inhibition of dopaminergic signaling via treatment with D1 or D2 receptor 
antagonists (Liu et al., 2012) and in D1 receptor knockout mice (Gallardo et al., 2014; Michalik et al., 2015). It has also been reported that mu-opioid receptor knockout mice display attenuated FAA when feeding is restricted to $3 \mathrm{~h}$ during the night (Kas et al., 2004), suggesting that endogenous opioids influence the robustness of FAA.

The current study supports the hypothesis that FAA is intricately linked to reward signaling. We found that wheelrunning activity enhanced FAA. The running wheel is rewarding to rodents and known to activate the dopaminergic system and influence endogenous opioids in their brains (Sherwin, 1998; Novak et al., 2012; Morgan et al., 2015). It has been shown that the rewarding effect of wheel running is influenced by endogenous opioids (Lett et al., 2001). The enhanced FAA we observed was not simply due to greater activity levels during wheel running because FAA was similarly enhanced by exposure to locked wheels, as long as the mice had been previously exposed to wheel-running. These data suggest that through operant conditioning, whereby the mice associate the intrinsically rewarding properties of running with the wheel, rewarding value is imparted on the locked wheel. Future studies should explore the putative link between FAA expression and the reward system via direct activation or inhibition of reward circuits.

Dopamine in the brain is also regulated by photoperiod in rodents. In short days, the number of hypothalamic tyrosine hydroxylase-positive neurons declined in male grass rats (Deats et al., 2015). In contrast, in long days, dopamine was elevated in the hypothalamus of C57BL/6J mice (Goda et al., 2015). Previously, our studies demonstrated that mice in long days had more robust FAA compared to short days (Figures 3, 4; Pendergast et al., 2009). A potential caveat of our initial finding was that the differences in phases of restricted feeding relative to the light-dark cycle in different photoperiods could account for the difference in FAA expression. In this study, we eliminated this caveat and showed that regardless of feeding time, FAA is less robust in short photoperiods compared to long photoperiods.

Recently, Dattolo et al. (2016) measured FAA in C57BL/6J mice with or without running discs. With running discs, mice had elevated FAA and reduced nighttime activity so they concluded there was a compensation effect (the total activity each day remained the same but was differentially distributed depending on the presence of the disc). In contrast, we found that both FAA and nocturnal activity were elevated upon exposing

\section{REFERENCES}

Angeles-Castellanos, M., Salgado-Delgado, R., Rodríguez, K., Buijs, R. M., and Escobar, C. (2008). Expectancy for food or expectancy for chocolate reveals timing systems for metabolism and reward. Neuroscience 155, 297-307. doi: 10. 1016/j.neuroscience.2008.06.001

Antle, M. C., and Silver, R. (2009). Neural basis of timing and anticipatory behaviors. Eur. J. Neurosci. 30, 1643-1649. doi: 10.1111/j.1460-9568.2009. 06959.x

Belke, T. W. (1997). Running and responding reinforced by the opportunity to run: effect of reinforcer duration. J. Exp. Anal. Behav. 67, 337-351. doi: 10.1901/jeab. 1997.67-337 mice to free wheels. However, FAA, but not nocturnal activity, was elevated in wheel-experienced mice during exposure to locked wheels. In both studies, passive IR detectors were used to measure general activity; therefore, we do not know what factors accounted for the different results. It is possible that running discs and running wheels have distinct effects on nocturnal activity.

Although FAA was originally described by Richter (1922), relatively little progress has been made in understanding its underlying mechanisms. This is in part due to the elusive nature of the food-entrainable oscillator that controls FAA (the anatomical locus of the food entrainable oscillator is unknown; Stephan, 2002; Davidson, 2009; Mistlberger, 2009). We do not know whether the putative dopaminergic reward signaling that causes the wheel-running enhancement of FAA directly modifies the food-entrainable oscillator itself or acts downstream of the oscillator by regulating its outputs. However, our study is another step toward elucidating the molecular and anatomical substrates of FAA.

\section{AUTHOR CONTRIBUTIONS}

SY conceived the experiments. DEFLF, CNB, LJ and SY designed and performed the experiments. DEFLF and SY analyzed the data. DEFLF, CNB and SY wrote the manuscript.

\section{FUNDING}

This research was supported by a National Science Foundation grant IOS-1419477 to SY. DEFLF was supported by Fundação de Amparo à Pesquisa do Estado de São Paulo (FAPESP) process 2013/24740-3.

\section{ACKNOWLEDGMENTS}

The authors thank Dr. Julie S. Pendergast for comments on the manuscript and Dr. André Frazão Helene for guidance regarding statistical analyses of the data.

\section{SUPPLEMENTARY MATERIAL}

The Supplementary Material for this article can be found online at: http://journal.frontiersin.org/article/10.3389/fnbeh. 2016.00143/abstract

Belke, T. W., and Wagner, J. P. (2005). The reinforcing property and the rewarding aftereffect of wheel running in rats: a combination of two paradigms. Behav. Processes 68, 165-172. doi: 10.1016/j.beproc.2004.12.006

Collier, G., and Hirsch, E. (1971). Reinforcing properties of spontaneous activity in the rat. J. Comp. Physiol. Psychol. 77, 155-160. doi: 10.1037/h00 31588

Dattolo, T., Coomans, C. P., van Diepen, H. C., Patton, D. F., Power, S., Antle, M. C., et al. (2016). Neural activity in the suprachiasmatic circadian clock of nocturnal mice anticipating a daytime meal. Neuroscience 315, 91-103. doi: 10.1016/j.neuroscience.2015.12.014

Davidson, A. J. (2009). Lesion studies targeting food-anticipatory activity. Eur. J. Neurosci. 30, 1658-1664. doi: 10.1111/j.1460-9568.2009.06961.x 
Deats, S. P., Adidharma, W., and Yan, L. (2015). Hypothalamic dopaminergic neurons in an animal model of seasonal affective disorder. Neurosci. Lett. 602, 17-21. doi: 10.1016/j.neulet.2015.06.038

DeCoursey, P. J. (2014). Survival value of suprachiasmatic nuclei (SCN) in four wild sciurid rodents. Behav. Neurosci. 128, 240-249. doi: 10.1037/a0036696

Flores, D. E., Bettilyon, C. N., and Yamazaki, S. (2016). Period-independent novel circadian oscillators revealed by timed exercise and palatable meals. Sci. Rep. 6:21945. doi: 10.1038/srep21945

Gallardo, C. M., Darvas, M., Oviatt, M., Chang, C. H., Michalik, M., Huddy, T. F., et al. (2014). Dopamine receptor 1 neurons in the dorsal striatum regulate food anticipatory circadian activity rhythms in mice. Elife 3:e03781. doi: 10. 7554/eLife.03781

Goda, R., Otsuka, T., Iwamoto, A., Kawai, M., Shibata, S., Furuse, M., et al. (2015). Serotonin levels in the dorsal raphe nuclei of both chipmunks and mice are enhanced by long photoperiod, but brain dopamine level response to photoperiod is species-specific. Neurosci. Lett. 593, 95-100. doi: 10.1016/j. neulet.2015.03.035

Hammer, Ø., Harper, D. A. T., and Ryan, P. D. (2001). Past: paleontological statistics software package for education and data analysis. Palaeont. Electr. 4, 1-9. Available online at: http://palaeo-electronica.org/2001_1/ past/past.pdf

Hsu, C. T., Dollar, P., Chang, D., and Steele, A. D. (2010a). Daily timed sexual interaction induces moderate anticipatory activity in mice. PLoS One 5:e15429. doi: 10.1371/journal.pone.0015429

Hsu, C. T., Patton, D. F., Mistlberger, R. E., and Steele, A. D. (2010b). Palatable meal anticipation in mice. PLoS One 5:e12903. doi: 10.1371/journal.pone. 0012903

Iijima, M., Yamaguchi, S., Van Der Horst, G. T., Bonnefont, X., Okamura, H., and Shibata, S. (2005). Altered food-anticipatory activity rhythm in cryptochromedeficient mice. Neurosci. Res. 52, 166-173. doi: 10.1016/j.neures.2005. 03.003

Iversen, I. H. (1993). Techniques for establishing schedules with wheel running as reinforcement in rats. J. Exp. Anal. Behav. 60, 219-238. doi: 10.1901/jeab.1993. 60-219

Izumo, M., Pejchal, M., Schook, A. C., Lange, R. P., Walisser, J. A., Sato, T. R., et al. (2014). Differential effects of light and feeding on circadian organization of peripheral clocks in a forebrain bmal1 mutant. Elife 3:e04617. doi: 10 . 7554/eLife.04617

Jansen, H. T., Sergeeva, A., Stark, G., and Sorg, B. A. (2012). Circadian discrimination of reward: evidence for simultaneous yet separable food- and drug-entrained rhythms in the rat. Chronobiol. Int. 29, 454-468. doi: 10. 3109/07420528.2012.667467

Johnson, C. H., Elliott, J. A., and Foster, R. (2003). Entrainment of circadian programs. Chronobiol. Int. 20, 741-774. doi: 10.1081/cbi-120024211

Kagan, J., and Berkun, M. (1954). The reward value of running activity. J. Comp. Physiol. Psychol. 47:108. doi: 10.1037/h0058877

Kas, M. J., van den Bos, R., Baars, A. M., Lubbers, M., Lesscher, H. M., Hillebrand, J. J., et al. (2004). Mu-opioid receptor knockout mice show diminished food-anticipatory activity. Eur. J. Neurosci. 20, 1624-1632. doi: 10. 1111/j.1460-9568.2004.03581.x

Keith, D. R., Hart, C. L., Robotham, M., Tariq, M., Le Sauter, J., and Silver, R. (2013). Time of day influences the voluntary intake and behavioral response to methamphetamine and food reward. Pharmacol. Biochem. Behav. 110, 117-126. doi: 10.1016/j.pbb.2013.05.011

Ko, C. H., and Takahashi, J. S. (2006). Molecular components of the mammalian circadian clock. Hum. Mol. Genet. 15, R271-R277. doi: 10.1007/978-3-64225950-0_1

Landry, G. J., Opiol, H., Marchant, E. G., Pavlovski, I., Mear, R. J., Hamson, D. K., et al. (2012). Scheduled daily mating induces circadian anticipatory activity rhythms in the male rat. PLoS One 7:e40895. doi: 10.1371/journal.pone. 0040895

Lehman, M. N., Silver, R., Gladstone, W. R., Kahn, R. M., Gibson, M., and Bittman, E. L. (1987). Circadian rhythmicity restored by neural transplant. Immunocytochemical characterization of the graft and its integration with the host brain. J. Neurosci. 7, 1626-1638.

Lett, B. T., Grant, V. L., and Koh, M. T. (2001). Naloxone attenuates the conditioned place preference induced by wheel running in rats. Physiol. Behav. 72, 355-358. doi: 10.1016/s0031-9384(00)00427-3
Liu, Y. Y., Liu, T. Y., Qu, W. M., Hong, Z. Y., Urade, Y., and Huang, Z. L. (2012). Dopamine is involved in food-anticipatory activity in mice. J. Biol. Rhythms 27, 398-409. doi: 10.1177/0748730412455913

Mather, J. G. (1981). Wheel-running activity - a new interpretation. Mammal Rev. 11, 41-51. doi: 10.1111/j.1365-2907.1981.tb00246.x

Meijer, J. H., and Robbers, Y. (2014). Wheel running in the wild. Proc. Biol. Sci. 281:20140210. doi: 10.1098/rspb.2014.0210

Mendoza, J., Angeles-Castellanos, M., and Escobar, C. (2005a). A daily palatable meal without food deprivation entrains the suprachiasmatic nucleus of rats. Eur. J. Neurosci. 22, 2855-2862. doi: 10.1111/j.1460-9568.2005. 04461.x

Mendoza, J., Angeles-Castellanos, M., and Escobar, C. (2005b). Differential role of the accumbens shell and core subterritories in food-entrained rhythms of rats. Behav. Brain Res. 158, 133-142. doi: 10.1016/j.bbr.2004.08.016

Mendoza, J., Angeles-Castellanos, M., and Escobar, C. (2005c). Entrainment by a palatable meal induces food-anticipatory activity and c-fos expression in reward-related areas of the brain. Neuroscience 133, 293-303. doi: 10.1016/j. neuroscience.2005.01.064

Mendoza, J., and Challet, E. (2014). Circadian insights into dopamine mechanisms. Neuroscience 282C, 230-242. doi: 10.1016/j.neuroscience.2014. 07.081

Michalik, M., Steele, A. D., and Mistlberger, R. E. (2015). A sex difference in circadian food-anticipatory rhythms in mice: interaction with dopamine D1 receptor knockout. Behav. Neurosci. 129, 351-360. doi: 10.1037/bne00 00058

Mistlberger, R. E. (1994). Circadian food-anticipatory activity: formal models and physiological mechanisms. Neurosci. Biobehav. Rev. 18, 171-195. doi: 10. 1016/0149-7634(94)90023-x

Mistlberger, R. E. (2009). Food-anticipatory circadian rhythms: concepts and methods. Eur. J. Neurosci. 30, 1718-1729. doi: 10.1111/j.1460-9568.2009. 06965.x

Mistlberger, R., and Rusak, B. (1987). Palatable daily meals entrain anticipatory activity rhythms in free-feeding rats: dependence on meal size and nutrient content. Physiol. Behav. 41, 219-226. doi: 10.1016/0031-9384(87) 90356-8

Mohawk, J. A., Pezuk, P., and Menaker, M. (2013). Methamphetamine and dopamine receptor D1 regulate entrainment of murine circadian oscillators. PLoS One 8:E62463. doi: 10.1371/journal.pone.0062463

Moore, R. Y., and Eichler, V. B. (1972). Loss of a circadian adrenal corticosterone rhythm following suprachiasmatic lesions in the rat. Brain Res. 42, 201-206. doi: 10.1016/0006-8993(72)90054-6

Morgan, J. A., Corrigan, F., and Baune, B. T. (2015). Effects of physical exercise on central nervous system functions: a review of brain region specific adaptations. J. Mol. Psychiatry 3:3. doi: 10.1186/s40303-015-0010-8

Novak, C. M., Burghardt, P. R., and Levine, J. A. (2012). The use of a running wheel to measure activity in rodents: relationship to energy balance, general activity, and reward. Neurosci. Biobehav. Rev. 36, 1001-1014. doi: 10.1016/j.neubiorev. 2011.12.012

Pendergast, J. S., Nakamura, W., Friday, R. C., Hatanaka, F., Takumi, T., and Yamazaki, S. (2009). Robust food anticipatory activity in BMAL1-deficient mice. PLoS One 4:E4860. doi: 10.1371/journal.pone.0004860

Pendergast, J. S., Oda, G. A., Niswender, K. D., and Yamazaki, S. (2012). Period determination in the food-entrainable and methamphetamine-sensitive circadian oscillator(s). Proc. Natl. Acad. Sci. U S A 109, 14218-14223. doi: 10. 1073/pnas.1206213109

Pitts, S., Perone, E., and Silver, R. (2003). Food-entrained circadian rhythms are sustained in arrhythmic Clk/Clk mutant mice. Am. J. Physiol. Regul. Integr. Comp. Physiol. 285, R57-R67. doi: 10.1152/ajpregu.00023.2003

Ralph, M. R., Foster, R. G., Davis, F. C., and Menaker, M. (1990). Transplanted suprachiasmatic nucleus determines circadian period. Science 247, 975-978. doi: $10.1126 /$ science. 2305266

Richter, C. P. (1922). A behavioristic study of the activity of the rat. Comp. Psychol. Monogr. 1, 1-54.

Rusak, B. (1977). The role of the suprachiasmatic nuclei in the generation of circadian rhythms in the golden hamster, Mesocricetus auratus. J. Comp. Physiol. A 118, 145-164. doi: 10.1007/bf00611819

Sawaki, Y., Nihonmatsu, I., and Kawamura, H. (1984). Transplantation of the neonatal suprachiasmatic nuclei into rats with complete bilateral 
suprachiasmatic lesions. Neurosci. Res. 1, 67-72. doi: 10.1016/0168-0102 (84)90031-2

Sherwin, C. M. (1998). Voluntary wheel running: a review and novel interpretation. Anim. Behav. 56, 11-27. doi: 10.1006/anbe.1998.0836

Sorg, B. A., Stark, G., Sergeeva, A., and Jansen, H. T. (2011). Photoperiodic suppression of drug reinstatement. Neuroscience 176, 284-295. doi: 10.1016/j. neuroscience.2010.12.022

Spoelstra, K., Wikelski, M., Daan, S., Loudon, A. S., and Hau, M. (2016). Natural selection against a circadian clock gene mutation in mice. Proc. Natl. Acad. Sci. U S A 113, 686-691. doi: 10.1073/pnas.1516442113

Stephan, F. K. (2002). The "other" circadian system: food as a zeitgeber. J. Biol. Rhythms 17, 284-292. doi: 10.1177/074873002129002591

Stephan, F. K., and Zucker, I. (1972). Circadian rhythms in drinking behavior and locomotor activity of rats are eliminated by hypothalamic lesions. Proc. Natl. Acad. Sci. U S A 69, 1583-1586. doi: 10.1073/pnas.69. 6.1583

Storch, K. F., and Weitz, C. J. (2009). Daily rhythms of food-anticipatory behavioral activity do not require the known circadian clock. Proc. Natl. Acad. Sci. U S A 106, 6808-6813. doi: 10.1073/pnas.0902063106

Verwey, M., Khoja, Z., Stewart, J., and Amir, S. (2007). Differential regulation of the expression of Period2 protein in the limbic forebrain and dorsomedial hypothalamus by daily limited access to highly palatable food in food-deprived and free-fed rats. Neuroscience 147, 277-285. doi: 10.1016/j.neuroscience.2007. 04.044
Webb, I. C., Baltazar, R. M., Lehman, M. N., and Coolen, L. M. (2009). Bidirectional interactions between the circadian and reward systems: is restricted food access a unique zeitgeber? Eur. J. Neurosci. 30, 1739-1748. doi: 10.1111/j.1460-9568.2009.06966.x

Winfree, A. T. (1980). The Geometry of Biological Time, (New York, NY: SpringerVerlag), p. 530.

Yamazaki, S., Numano, R., Abe, M., Hida, A., Takahashi, R., Ueda, M., et al. (2000) Resetting central and peripheral circadian oscillators in transgenic rats. Science 288, 682-685. doi: 10.1126/science.288.5466.682

Yoo, S. H., Yamazaki, S., Lowrey, P. L., Shimomura, K., Ko, C. H., Buhr, E. D., et al. (2004). PERIOD2::LUCIFERASE real-time reporting of circadian dynamics reveals persistent circadian oscillations in mouse peripheral tissues. Proc. Natl. Acad. Sci. U S A 101, 5339-5346. doi: 10.1073/pnas.0308709101

Conflict of Interest Statement: The authors declare that the research was conducted in the absence of any commercial or financial relationships that could be construed as a potential conflict of interest.

Copyright (C) 2016 Flôres, Bettilyon, Jia and Yamazaki. This is an open-access article distributed under the terms of the Creative Commons Attribution License (CC BY). The use, distribution and reproduction in other forums is permitted, provided the original author(s) or licensor are credited and that the original publication in this journal is cited, in accordance with accepted academic practice. No use, distribution or reproduction is permitted which does not comply with these terms. 